\title{
A RARE CASE OF RUPTURED ANEURYSM AT THE ORIGIN OF INFERIOR PART OF DUPLICATED MIDDLE CEREBRAL ARTERY
}

\author{
Achmad Firdaus Sani ${ }^{1}$, Dedy Kurniawan ${ }^{1}$ \\ Correspondence: dedy_k2002@yahoo.com \\ ${ }^{I}$ Division of Neurointervention, Department of Neurology, Airlangga University/ Dr. Soetomo Academic Medical Center Hospital \\ Surabaya Indonesia
}

\author{
Article History: \\ Received: September 2, 2020 \\ Accepted: June 28, 2021 \\ Published: July 1, 2021 \\ Cite this as: \\ Sani AF, Kurniawan D. A rare \\ case of ruptured aneurysm at the \\ origin of inferior part of \\ duplicated middle cerebral \\ artery. Malang Neurology \\ Journal; 2021.7: 146-148. \\ http://dx.doi.org/10.21776/ub.mnj \\ .2021.007.02.13
}

\begin{abstract}
Duplicated middle cerebral artery (DMCA) is an anomalous vessel arises from the internal carotid artery (ICA). This anatomical variation is rare. Aneurysm with this anatomical variation and unusual form was very rare. Even though this kind of aneurysm is rare, it was often ruptured. In this paper, we report a case of 40-years old female with abrupt decreased of consciousness as a chief complaint, along with severe headache one day earlier, no history of head trauma, and there was nuchal rigidity. She didn't had history of hypertension before. Head computed tomography showed subarachnoid hemmorrhage (SAH) mostly on the left sylvian fissure with Hunt and Hess scale was 3 and Fisher scale was 2 , while the cerebral angiography showed duplication of the left middle cerebral artery in which the inferior part of the MCA duplication has ruptured aneurysm at the origin. Treatment option for this aneurysm is endovascular coiling with preserved of the inferior part of duplicated MCA. Result of this treatment shows a good outcome.
\end{abstract}

Keywords: Duplicated middle cerebral artery, ruptured aneurysm, coiling

\section{Introduction}

Anatomical variation in the middle cerebral artery (MCA) are rare and classified into : duplicated MCA (DMCA), an accessory MCA, and fenestration of $\mathrm{MCA}^{1,2,3,4}$. The incidence of DMCA has been reported to be $0,7-2,9 \%$ on autopsy ${ }^{4}, 0,81 \%$ on magnetic resonance angiography $(\mathrm{MRA})^{3}$ and $0,24-1.5 \%$ on angiography ${ }^{4,5}$. We report a case of ruptured aneurysm at the origin of inferior part of duplicated middle cerebral artery. The aneurysm also has unusual "heart shaped" form.

\section{Case Report}

The A 40 years old female came to our hospital with abrupt decreased of consciousness as a chief complaint and a severe headache one day earlier with no history of head trauma. She didn't had history of hypertension. Neurological examination on admission, the Glasgow Coma Scale (GCS) was 8 with nuchal rigidity. Other neurological examination showed no abnormality. Head computed tomography showed subarachnoid hemorrhage mostly on left Sylvian fissure (Figure 1), with Hunt and Hess scale was 3, Fisher scale was 2. Cerebral angiography showed duplication of the left MCA. Inferior part of the MCA duplication arised from internal carotid artery and the aneurysm was at the origin. The aneurysmal neck was 1,1 $\mathrm{mm}$ and dome was $3,7 \mathrm{~mm} \times 3,7 \mathrm{~mm}$. It was pointing anterolateral. The aneurysm has " heart-shaped" form
(Figure 2). There are no abnormalities at the right carotid and vertebrobasilar system.

Coiling aneurysm was performed using guiding catheter Chaperon 6 Fr, microcatheter Echelon 10 over micro guidewire hybrid 1214, with 2 target coils were inserted that were $3 \mathrm{~mm} \times 6 \mathrm{~cm} \mathrm{3D}$ and $2 \mathrm{~mm} \times 6 \mathrm{~cm}$ helical. The coiling was successful and confirmed with cerebral angiography (Figure 2). She was discharged one week after the procedure with GCS 15 and no neurological deficit.

\section{Discussion}

DMCA is an anomalous vessel arising from the internal carotid artery (ICA) ${ }^{6}$ Komiyama et al, reported that a duplicated MCA is an anomalistic embryologically early ramification of the early branch of the MCA which originates from the distal end of the $\mathrm{ICA}^{7}$. There are two theories for course and the cortical territorry of DMCA. First theory said that the DMCA can be considered as an early temporal branch of the MCA. Meanwhile, second theory said that DMCA emerge as the direct bifurcation of the main trunk $\mathrm{MCA}^{6}$. There were two types for the classification of DMCA, type A and B. Type A refers to cases in which the duplication of the middle cerebral artery separates from the ICA bifurcation while type B refers to cases in which the blood vessels arise from the ICA between the anterior choroidal artery (AChA) and bifurcation of the internal carotid artery ${ }^{4}$. Kai et al reported that all aneurysms were found at type B DMCA can be 

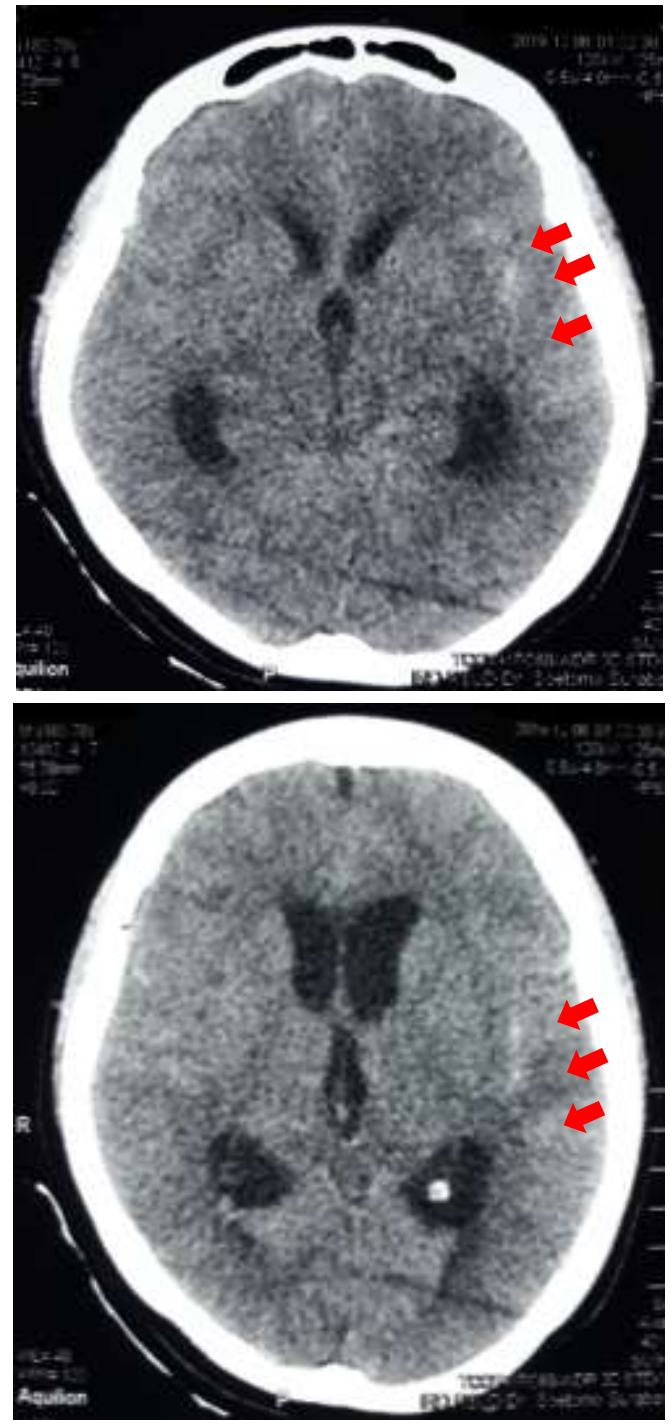

Figure 1 . Head non contrast CT scan axial view. It showed hyperdensity on subarachnoid space, and markedly on left slyvian fissure ( red arrow ), also seen early dilatation of the lateral ventricle.

expected to have higher hemodynamic stress and is a factor of development aneurysms on it $^{6}$

Incidence of DMCA is rare, both DMCA and also the aneurysm at the origin of the DMCA is very rare $^{8}$. Koyama et al also reported DMCA with aneurysm on the right side to occur more often than on the left side. The probability is that cases on the left side was two from nine cases ${ }^{9}$. An aneurysm at the DMCA origin was initially reported by Crompton and Lond in $1962^{10,11}$. Duplication of intracranial arteries have been associated with the development of brain aneurysm $^{12}$. There were many etiological factors proposed for the aneurysm. Congenital factors which occur in cerebrovascular development such as congenital defects of the media, residual fetal arteries, and maldevelopment at the fetal stage. Acquired factors, such as degenerative process of the vessels, arteriosclerotic process and hemodynamic stress may also be involved ${ }^{13,14}$. Cerebral aneurysm with "heart-shaped" form is interesting and extremely rare and is usually associated with vessel-wall degeneration and hemodynamic stress. In this report, DMCA can affect the hemodynamic state and is related with the rupture of the aneurysm incidence at the site where the DMCA originated ${ }^{12,15}$.
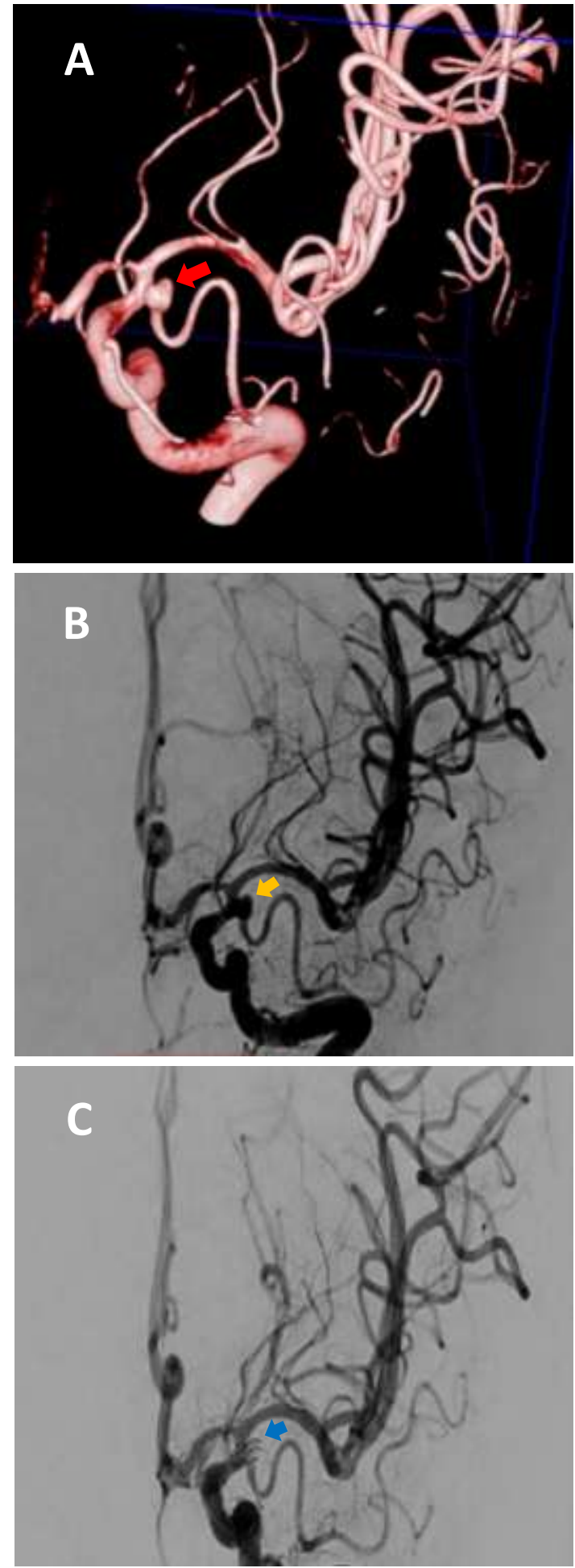

Figure 2. The aneurysm located at origin of inferior part of DMCA with " heart-shaped " configuration ( red arrow ) (A), the aneurysm before coiling (yellow arrow) (B), and the aneurysm after coiling ( blue arrow ) (C), the inferior part of DMCA remain patent.

This case, from the patient's history taking, there was severe headache followed by decreased of counsciousness abruptly and no history of trauma that could be a secondary headache ${ }^{16}$. It was more describing a spontaneous SAH in which the majority spontaneous SAH caused by ruptured aneurysm so urgent treatment was needed to obliterate the aneurysm and avoid the increased risk of mortality ${ }^{17}$. Treatment option for this aneurysm are endovascular procedure and open surgery. For the treatment of 
aneurysms at the origins of the DMCA, open surgery is reported to be opted more frequently than endovascular procedure. Neverthelles, both treatment options actualy attain a good result ${ }^{12}$.In our case, It was a case with left type B DMCA that has the aneurysm at the origin of inferior part of DMCA. The diameter DMCA was almost the same as that of the main MCA. We treated this aneurysm with endovascular procedure to prevent re-rupture. Another thing needs to be highlighted here is that the DMCA plays an important role in supplying collateral blood flow to the frontal lobe, anterior temporal lobe and basal ganglia through the perforating arteries. ${ }^{2,7,18}$ Cerebral angiography evaluation after coiling confirmed that the inferior part of MCA duplication was patent. Patient was discharged without any neurological deficit.

\section{Conclusion}

The case of DMCA with aneurysm at the origin of inferior part is rare. However, the incident of rupture or re-rupture of aneurysm is high because there are several factors affect it. In this case, coiling aneurysm showed to be a good choice of treatment with preserved inferior part of MCA duplication.

\section{Acknowledgement}

None.

\section{Conflict of Interest}

The authors declare that there is no conflict of interest.

\section{References}

1. Laborde DV, Mason AM, Riley J, Dion JE, Barrow DL. Aneurysm of a duplicate middle cerebral artery. World Neurosurg; 2012. 77(1):201.e1-201.e4. DOI:10.1016/j.wneu.2011.03.038

2. Uchiyama N. Anomalies of the middle cerebral artery. Neurol Med Chir (Tokyo); 2017. 57(6):261-266. DOI:10.2176/nmc.ra.2017-0043

3. Kim MS, Hur JW, Lee JW, Lee HK. Middle cerebral artery anomalies detected by conventional angiography and magnetic resonance angiography. J Korean Neurosurg SocSoc; 2005. Avalaible from: https://www.jkns.or.kr/journal/view.php?number=211 9

4. Stojanović NN, Kostić A, Mitić R, Berilažić L. Correlation between multiple cerebral aneurysms and a rare type of segmental duplication of the middle cerebral artery. BMC Neurol; 2020. 20(1):1-4. DOI:10.1186/s12883-019-1588-8

5. Kim JS, Lee C-H, Park H, Han J-W. An Unruptured Cerebral Aneurysm at the Origin of the Duplicated Middle Cerebral Artery. J Cerebrovasc Endovasc Neurosurg; 2015. 17(3):223.

DOI:10.7461/jcen.2015.17.3.223

6. Chang HY, Kim MS. Middle cerebral artery duplication: Classification and clinical implications. J Korean Neurosurg Soc; 2011. 49(2):102-106. DOI:10.3340/jkns.2011.49.2.102
7. Komiyama M, Nakajima H, Nishikawa M, Yasui T. Middle cerebral artery variations: Duplicated and accessory arteries. Am J Neuroradiol; 1998. 19(1):4549. Avalaible

from:

https://pubmed.ncbi.nlm.nih.gov/9432156/

8. Imahori T, Mizobe T, Fujinaka T, et al. An aneurysm at the origin of a duplicated middle cerebral artery treated by stent-assisted coiling using the "WrappedCandy" Low-Profile Visualized Intraluminal Support (LVIS) technique: A technical case report and review of the literature. World Neurosurg; 2020. 143:353359. DOI:10.1016/j.wneu.2020.08.046

9. Koyama S, Kotani A, Sasaki J, Tazoe M, Tsubokawa T. Ruptured Aneurysm at the Origin of Duplication of the Middle Cerebral Artery: Case report. Neurol Med Chir (Tokyo); 1995. 35(9):671-673.

DOI: $10.2176 /$ nmc.35.671

10. Crompton MR. The pathology of ruptured middlecerebral aneurysms with special reference to the differences between the sexes. Lancet; 1962. 280(7253):421-425.

DOI: $10.1016 / \mathrm{S} 0140-6736(62) 90281-7$

11. Iida Y, Tamase A, Kamide T, Mori K, Seki S, Nomura M. Aneurysm at origin of duplicated middle cerebral artery associated with another aneurysm. Surg Neurol Int; 2015. 6:S549-S552. DOI:10.4103/2152-7806.168069

12. Ren H, Ma L, Wei M, Li J, Yu M, Yin L. Duplicated middle cerebral artery origin with an aneurysm. Med (United States); 2018. 97(9):3-6. DOI: 10.1097/MD.0000000000009947

13. Hori E, Kurosaki K, Matsumura N, et al. Multiple aneurysms arising from the origin of a duplication of the middle cerebral artery. J Clin Neurosci; 2005. 12(7):812-815. DOI: 10.1016/j.jocn.2004.08.033

14. Hou K, Xu K, Liu H, Li G, Yu J. The Clinical characteristics and treatment considerations for intracranial aneurysms associated with middle cerebral artery anomalies: A systematic review. Front Neurol; 2020. 11(October).

DOI: $10.3389 /$ fneur.2020.564797

15. Choi J, Joo S, Lee J, Kim T. Middle cerebral artery variations associated with intracranial aneurysmal rupture. J Korean Neurosurg Soc; 2006. 467-470. Avalaible

from: koreascience.or.kr/article/JAKO200608410671346.pd $\mathrm{f}$

16. Hidayati HB. The Clinician's Approach to The Management of Headache. Malang Neurol Journal; 2016. 02. DOI:10.21776/ub.mnj.2016.002.02.7

17. Ramadhania NN, Darmawan AF, Sani AF. Higher mean arterial pressure increases risk of in-hospital mortality in aneurysmal subarachnoid hemorrhage. $\begin{array}{lll}\text { Universa } & \text { Med; } & 2020 .\end{array}$ DOI:10.18051/univmed.2020.v39.153-161

18. Otani N, Nawashiro H, Tsuzuki N, et al. A ruptured internal carotid artery aneurysm located at the origin of the duplicated middle cerebral artery associated with accessory middle cerebral artery and middle cerebral artery aplasia. Surg Neurol Int; 2010. 1(1):51. DOI:10.4103/2152-7806.69378 